\title{
AN EFFICIENT METHOD FOR THE CALCULATION OF MEAN EXTINCTION. I. THE ANALYTICITY OF THE COMPLEX EXTINCTION EFFICIENCY OF HOMOGENEOUS SPHERES
}

\author{
Zhang-Fan Xing and J. MaYo Greenberg \\ Leiden University, Laboratory Astrophysics, Postbus 9504, 2300 RA Leiden, The Netherlands \\ Received 1992 January 28; accepted 1992 March 2
}

\begin{abstract}
The analyticity of the complex extinction efficiency $\widetilde{Q}_{\text {ext }}$ has been investigated in different parameter domains. From the concept of causality, it follows that, in the angular frequency $\omega$ domain, $\widetilde{Q}_{\text {ext }}$ for arbitrary finite particles must be analytic in the entire lower complex $\tilde{\omega}$-plane. While, at fixed frequency, the extinction efficiency approaches "paradoxically" the limit 2 for large particle size $a$, it is noted that $Q_{\text {ext }}$ must approach 0 at high frequency for certain physical reasons. In the size parameter domain, $x=\omega a / c$, numerical Hilbert transforms are used to study the analyticity properties of $\widetilde{Q}_{\text {ext }}$ for homogeneous spheres. It is found that $\widetilde{Q}_{\text {ext }}$ is analytic in the entire lower complex $\tilde{x}$-plane when the refractive index $m$ is fixed as a real constant (pure scattering) or $\infty$ (perfect conductor), but that poles appear in the left side of the lower complex $\tilde{x}$-plane as $m$ becomes complex. These properties of the complex extinction efficiency have been shown to result in a powerful approximation method. The computation of the mean extinction produced by an extended size distribution of particles may be conveniently and accurately approximated using only a few values of the complex extinction evaluated in the complex plane.
\end{abstract}

Subject headings: dust, extinction - methods: analytical - methods: numerical

\section{INTRODUCTION}

The extinction efficiency $Q_{\text {ext }}$ (extinction cross section divided by geometrical cross section) is a fundamental quantity needed in many applications. Since Mie did his pioneer work almost 100 years ago, various exact and approximate methods have been devised to obtain this important factor for nonspherical and inhomogeneous particles as well as for spheres (van de Hulst 1957; Kerker 1969; Bohren \& Huffman 1983; Purcell \& Pennypacker (1973); Asano \& Yamamoto 1975; Hage, Greenberg, \& Wang 1991). For the past three decades this study has been greatly advanced due to the fast development of computer technology. Programs for simple particles like spheres and cylinders are easily handled on a personal computer. However, for more complicated cases (spheroids, porous particles, etc.), the scattering problem is still relatively intractable and requires larger computers for fast and accurate evaluation of $Q_{\text {ext }}$. There exists a strong and continuing demand for discovering more and more efficient algorithms.

In most applications we are confronted with the problem of the extinction produced not by a single particle but by a cloud of particles of various sizes, shapes, morphologies, and material constituents. Here we shall only consider the cases of single scattering produced by independent particles. If the particles can be parameterized by size alone as for spheres, the mean extinction cross section of this cloud will be

$$
\bar{C}_{\mathrm{ext}}=\int_{0}^{\infty} C_{\mathrm{ext}}(k a) n(a) d a,
$$

where $k=2 \pi / \lambda$ is the wavenumber, $\lambda$ is the wavelength of the incident light in vacuum; $a$ denotes a linear measure of the particle size (for spheres this is the radius); $C_{\text {ext }}(k a)$ is the extinction cross section at a specified size $a ; n(a)$ represents the normalized particle size distribution in the cloud. This integral is usually obtained by summing $C_{\text {ext }}(k a) n(a)$ over many discrete particle sizes. For complicated particle configurations, such a computation may become extremely time-consuming. Much more efficient approaches are needed.

It would be extremely useful if one could apply to equation (1) the mathematical tool for evaluating an infinite integral by replacing it with an appropriate contour integral which is simply calculated if the integrand has only simple poles. Going further, if the complex extinction efficiency $\widetilde{Q}_{\text {ext }}$ has certain analyticity properties (this will be fully addressed in the coming sections) and the size distribution $n(a)$ may be approximated by appropriate functions with only simple poles in the complex plane (see $\S 5$ ), the well-known residue theorem will lead us to a considerable simplification; that is, the integral in equation (1) may be alternatively evaluated by calculating the sum of the residues at discrete poles. Consequently the CPU time required will be greatly reduced. The validity of such an approximation relies completely on the analyticity properties of $\tilde{Q}_{\text {ext }}$. The exploration of this analyticity is the main theme of our studies presented in this and subsequent papers.

Our inquiry into the analyticity was also motivated by a certain aesthetic curiosity. It has been widely known that particle scattering (whether classical or quantum mechanical) is a causal process (Newton 1966; Nussenzveig 1972). Consequently the frequency-dependent complex extinction efficiency satisfies dispersion relations which imply special analyticity properties in the complex frequency plane (see $\S 3$ ). In general the extinction efficiency is determined by a few parameters. For homogeneous spheres, this parameter dependence may be functionally written as

$$
Q_{\mathrm{ext}}=Q_{\mathrm{ext}}(m, x),
$$

where the refractive index $m=m(\omega)$ is a function of light frequency $\omega$ and the size parameter $x=\omega a / c$ depends on both radius $a$ and frequency $\omega$. $Q_{\text {ext }}$ can be considered as either a function of $\omega$ for fixed $a$ or a function of $a$ for fixed $\omega$. It is clear that parameters $\omega$ and $a$ affect $Q_{\text {ext }}$ in unsymmetric ways. Our question is this: how different will the analyticity properties be 
if the complex extinction efficiency is examined in different parameter domains?

Throughout our investigation, the Titchmarsh theorem plays a fundamental role (it will be introduced in $\S 2$; further explanations and some necessary clarifications are also presented there). Equipped with this basic theorem, we point out that two alternative approaches exist for studying the analyticity. One way is to examine the possible causality of the problem in hand. This is exemplified in $\S 3$ by analyzing the complex extinction efficiency in the angular frequency domain. The other uses Hilbert transforms computationally. Directly applying numerical transforms to the functions under investigation, one can infer their analyticity behavior from the differences between the transformed and corresponding untransformed functions. In $\S 4$ we apply this method to the complex extinction efficiency for the homogeneous spherical case, with the aim of understanding its analyticity in the particle size parameter domain. Based on the knowledge obtained in such a way, an approximation procedure is formulated in $\S 5$ which leads to a simplification in the calculation of the integral in equation (1). A range of computational examples are presented which validate our approximating method as an efficient and accurate one. In the last section our main results are outlined and further applications are indicated.

The convention and notation to be used in this paper will follow van de Hulst (1957), especially in choosing $e^{i \omega t}$ as the time-dependent factor of the plane-wave. Furthermore, the tilde is used to denote the complex extensions of the corresponding real quantities. For instance, $\tilde{\omega}$ is the complex extension of real $\omega$.

\section{TITCHMARSH THEOREM: ANALYTICITY, CAUSALITY, AND HILBERT TRANSFORMS}

In his classical book on the theory of the Fourier integral, Titchmarsh (1937) proved a theorem which connects analyticity, causality, and Hilbert transforms. According to this theorem, we have the following three equivalent statements for a complex function $F(x),{ }^{1}$ which is square integrable over the real $x$-axis.

1. Analyticity.-Replacing $x$ by its complex extension $\tilde{x}=$ $x+i y$, the function $F(\tilde{x})$ is analytic in the lower complex $\tilde{x}$-plane $(y<0)$ and approaches $F(x)$ almost everywhere as $y \rightarrow 0^{-}$. Furthermore, these exists a finite constant $K$ such that,

$$
\int_{-\infty}^{+\infty}|F(x+i y)|^{2} d x<K \text { for } y<0 .
$$

2. Causality.-The Fourier transform $f(t)$ of $F(x)$ vanishes for $t<0$, that is,

$$
f(t)=\frac{1}{\sqrt{2 \pi}} \int_{-\infty}^{+\infty} F(x) e^{i x t} d x=0 \quad(t<0) .
$$

3. Hilbert Transforms.-The real and imaginary parts of $F(x)$ are Hilbert transforms of each other:

$$
\begin{aligned}
& \operatorname{Re}\left[F\left(x_{0}\right)\right]=-\frac{1}{\pi} P \int_{-\infty}^{+\infty} \frac{\operatorname{Im}[F(x)]}{x-x_{0}} d x, \\
& \operatorname{Im}\left[F\left(x_{0}\right)\right]=+\frac{1}{\pi} P \int_{-\infty}^{+\infty} \frac{\operatorname{Re}[F(x)]}{x-x_{0}} d x,
\end{aligned}
$$

where the capital letter $P$ denotes the Cauchy principal value.

\footnotetext{
${ }^{1}$ In this section both $x$ and $t$ denote only the generic mathematical real variables. One should not confuse them with the size parameter, $x=2 \pi a / \lambda$, and the physical time $t$ as being used in the following sections.
}

Obviously, besides the analyticity, the first statement also requires $F(x)$ to be bounded in the lower complex plane. For all the cases we will consider in the present study, where mathematical strictness is not our major concern, it should be sufficient to replace the requirement on the boundedness of $F(x)$ by the following asymptotic property

$$
F(x)=O\left(\frac{1}{x}\right), \quad|x| \rightarrow \infty .
$$

The second statement is a mathematical formulation of one of the most important physical concepts-causality. This becomes obvious if we establish the following correspondences in equation (3): $t \leftrightarrow$ physical time and $x \leftrightarrow$ light angular frequency. It is sometimes hard to define causality precisely, but the general idea is illustrated as follows: the response (output) of a linear passive physical system cannot precede the cause (input). In the case of light scattering, it is that the scattered wave cannot be emitted by the scattering center before the incident wave has arrived. This subject has been extensively addressed in many research fields (Bode 1945; van Kampen 1953; Toll 1956; Nussenzveig 1972). In fact, causality is now recognized as being one of the essential requirements for any proposed physical theory to be accepted.

As Hilbert originally demonstrated, integrals (4a) and (4b) actually imply each other. Thus it suffices to keep only one of them. Nussenzveig (1972) referred to them as Plemelj formulas in recognition of their earlier origins. We will use the more customary designation and refer to both as Hilbert transforms. But there is one thing that we would like to clarify. Although they resemble each other, we think that it will be profitable to distinguish Hilbert transforms from dispersion relations. It is only when the complex function $F(x)$ satisfies the symmetry relation, or crossing condition,

$$
F(-x)=F^{*}(x),
$$

where the asterisk denotes the complex conjugate, that Hilbert transforms are equivalent to the ordinarily used dispersion relations, known in optics as Kramers-Kronig relations:

$$
\begin{aligned}
& \operatorname{Re}\left[F\left(x_{0}\right)\right]=-\frac{2}{\pi} P \int_{0}^{\infty} \frac{x \operatorname{Im}[F(x)]}{x^{2}-x_{0}^{2}} d x, \\
& \operatorname{Im}\left[F\left(x_{0}\right)\right]=+\frac{2}{\pi} P \int_{0}^{\infty} \frac{x_{0} \operatorname{Re}[F(x)]}{x^{2}-x_{0}^{2}} d x .
\end{aligned}
$$

The symmetry relation (6) is a consequence of requiring $f(t)$ in equation (3), the Fourier transform of $F(x)$, to be a real function. This is justifiable in the study of physical systems in the time domain, since any physical signal is always a real function of time. But from the mathematical point of view, $f(t)$ need not be real, if $t$ denotes a generic variable other than physical time; $f(t)$ may be a complex function and still fulfill the condition in statement (2) above.

The Titchmarsh theorem offers two options for studying the analyticity of $F(\tilde{x})$. In one way we can examine the possible causality of the problem. Another way is to check if the real and imaginary parts of $F(x)$ obey Hilbert transforms (4), which is especially helpful when we lack the required physical intuition for examining the problem under consideration. In the next section we shall follow the traditional approach of causality for studying the properties of the complex extinction efficiency in the angular frequency domain. However, in turning to the question of the analyticity in the particle size parameter domain in $\S 4$, we have been forced to use Hilbert 
transforms. Although this approach is a numerical one, it appears to provide a very powerful alternative to an analytical calculation.

\section{ANALYTICITY OF THE COMPLEX EXTINCTION EFFICIENCY}

\section{IN THE ANGULAR FREQUENCY DOMAIN}

Let us, for simplicity, consider the scalar scattering produced by a finite particle with arbitrary shape. Assume the particle is fixed in space and illuminated by a unit plane wave of angular frequency $\omega$, which propagates along the positive $z$-direction,

$$
u_{0}(\omega)=e^{-i k z+i \omega t}
$$

Following van de Hulst's argument (1957), the amplitude of the scattered wave at a distant field point $(r, \theta, \varphi)$ is

$$
u(\omega)=S(\omega, \theta, \varphi) \frac{e^{-i k r+i k z}}{i k r} u_{0}(\omega)
$$

where $r, \theta, \varphi$ are conventional spherical coordinates, wavenumber $k=\omega / c, c$ the speed of light in vacuum, and $S(\omega, \theta, \varphi)$ is the complex amplitude function. In the forward direction $\theta=0^{\circ}$, equation (8) becomes

$$
u(\omega)=\frac{S\left(\omega, 0^{\circ}\right)}{i(\omega / c) z} u_{0}(\omega)
$$

On the other hand, the frequency-dependent extinction efficiency $Q_{\text {ext }}(\omega)$ is related to the forward amplitude function $S(\omega$, $0^{\circ}$ ) by the well-known optical theorem

$$
Q_{\text {ext }}(\omega)=\frac{4 \pi}{(\omega / c)^{2} G} \operatorname{Re}\left[S\left(\omega, 0^{\circ}\right)\right] .
$$

Here $G$ denotes the geometrical area projected by the particle in the incident direction. We shall generalize equation (10) by introducing the complex extinction efficiency

$$
\tilde{Q}_{\text {ext }}(\omega)=\frac{4 \pi}{(\omega / c)^{2} G} S\left(\omega, 0^{\circ}\right) .
$$

A bit of derivation based on equations (9) and (11) leads to

$$
u(\omega)=C\left[i \omega \widetilde{Q}_{\mathrm{ext}}(\omega)\right] u_{0}(\omega),
$$

where $C=-G / 4 \pi c z$ is a real constant.

Clearly the complex function $\left[i \omega \widetilde{Q}_{\text {ext }}(\omega)\right]$ in equation (12) relates the input $u_{0}(\omega)$ to the output $u(\omega)$ in a linear and causal way. Additionally, the reality of the light signal in the physical time domain requires that $\left[i \omega \tilde{Q}_{\text {ext }}(\omega)\right]$ satisfies the symmetry relation (6). Hence, one may expect that the complex extinction efficiency $\widetilde{Q}_{\text {ext }}(\omega)$ will "potentially" fulfill the Kramers-Kronig relations (7a) and (7b). The reason for saying "potentially" is that until now the boundedness of $\widetilde{Q}_{\text {ext }}(\omega)$ as required by Titchmarsh theorem has not yet been checked. In the spirit of equation (5), this could be briefly demonstrated as follows.

At extremely high angular frequency (much higher than any resonant frequency), the electromagnetic response of any physically real material is essentially free electron-like. We can use the Lorentz model to determine the asymptotic behavior of its optical properties. Explicitly it is

$$
\epsilon(\omega)-1=m^{2}(\omega)-1=O\left(\frac{1}{\omega^{2}}\right), \quad|\omega| \rightarrow \infty,
$$

where $\epsilon(\omega)$ is the dielectric function of particle material and $m(\omega)$ the index of refraction. For simplicity, we shall only examine the case of spheres in the following. From the asymptotic behavior of $m(\omega)$ displayed above, it is easy to show that the phase shift $\rho(\omega)$, along the diameter of a sphere of radius $a$, will have the limit behavior

$$
\rho(\omega)=2[m(\omega)-1] x(\omega)=O\left(\frac{1}{\omega}\right), \quad|\omega| \rightarrow \infty .
$$

Here the dependence of the size parameter $x(\omega)=\omega a / c$ on the frequency $\omega$ is clarified.

Since now $|m(\omega)-1| \rightarrow 0,|\rho(\omega)| \rightarrow 0$, and $|x(\omega)| \rightarrow \infty$, when $|\omega| \rightarrow \infty$, we are actually in the adjoining boundary region " 12 " on the " $m-x$ domain" diagram (van de Hulst 1957, p. 132). In such a case the complex extinction efficiency is

$$
\begin{aligned}
\tilde{Q}_{\mathrm{ext}}(\omega) & =\frac{4}{x^{2}(\omega)} S\left(\omega, 0^{\circ}\right)=\frac{8}{3} i[m(\omega)-1] x(\omega) \\
& =\frac{4}{3} \rho(\omega) i=O\left(\frac{1}{\omega}\right), \quad|\omega| \rightarrow \infty
\end{aligned}
$$

Although the discussion above was made only for spheres, the asymptotic behavior (13) holds for particles of other types (Greenberg 1960).

Combining equation (13) with the discussions following equation (12), we see that $\widetilde{Q}_{\text {ext }}(\omega)$ must satisfy the KramersKronig relations, that is,

$$
\begin{aligned}
& \operatorname{Re}\left[\tilde{Q}_{\mathrm{ext}}\left(\omega_{0}\right)\right]=-\frac{2}{\pi} P \int_{0}^{\infty} \frac{\omega \operatorname{Im}\left[\tilde{Q}_{\mathrm{ext}}(\omega)\right]}{\omega^{2}-\omega_{0}^{2}} d \omega, \\
& \operatorname{Im}\left[\tilde{Q}_{\mathrm{ext}}\left(\omega_{0}\right)\right]=+\frac{2}{\pi} P \int_{0}^{\infty} \frac{\omega_{0} \operatorname{Re}\left[\tilde{Q}_{\mathrm{ext}}(\omega)\right]}{\omega^{2}-\omega_{0}^{2}} d \omega .
\end{aligned}
$$

As a consequence of the Titchmarsh theorem, $\tilde{Q}_{\text {ext }}(\tilde{\omega})$ will be analytic in the lower complex $\tilde{\omega}$-plane. Clearly these results are valid for any finite particles in any particular orientation so long as the scalar scattering theory applies.

It should be noted that $\mathrm{Ku} \&$ Felske (1986) arrived at the same dispersion relations as equation (14) for a different purpose. In that paper they thoroughly discussed both the lowand high-frequency asymptotic behaviors of semiconductor particles. It is also worth noting that there exists another approach for the analysis of the dispersive properties of $\widetilde{Q}_{\text {ext }}(\omega)$, namely, treating the cloud of particles as a formal linear causal medium (Purcell 1969).

From equation (13) we see that the asymptotic behavior of the real extinction efficiency $Q_{\text {ext }}(\omega)$ at large angular frequency $\omega$ is different from that obtained by ordinary diffraction theory, which basically deals with the approximation in the region of large particle size. Instead of approaching the value 2 , $Q_{\text {ext }}(\omega)$ must vanish at very high frequency for distinct physical reasons, that is,

$$
\lim _{|\omega| \rightarrow \infty} \tilde{Q}_{\mathrm{ext}}(\omega)=0
$$

As an illustration of the general behavior of the frequencydependent extinction efficiency, we present in Figure 1 the plots of $\widetilde{Q}_{\text {ext }}(\omega)$ for a homogeneous sphere. For such a sphere with radius $a, \widetilde{Q}_{\text {ext }}$ is directly determined by two parameters: size parameter $x=2 \pi a / \lambda=\omega a / c$ and complex refractive index $m(\omega)$. The light frequency $\omega$ affects $\widetilde{Q}_{\text {ext }}$ through both $x$ and $m(\omega)$. For simplicity of presentation, we have chosen $a=c$, so that $x=\omega$, and we can use $x$ and $\omega$ interchangably. In Figure 1a $\widetilde{Q}_{\text {ext }}(\omega)$ is shown for the frequency-dependent index of re- 

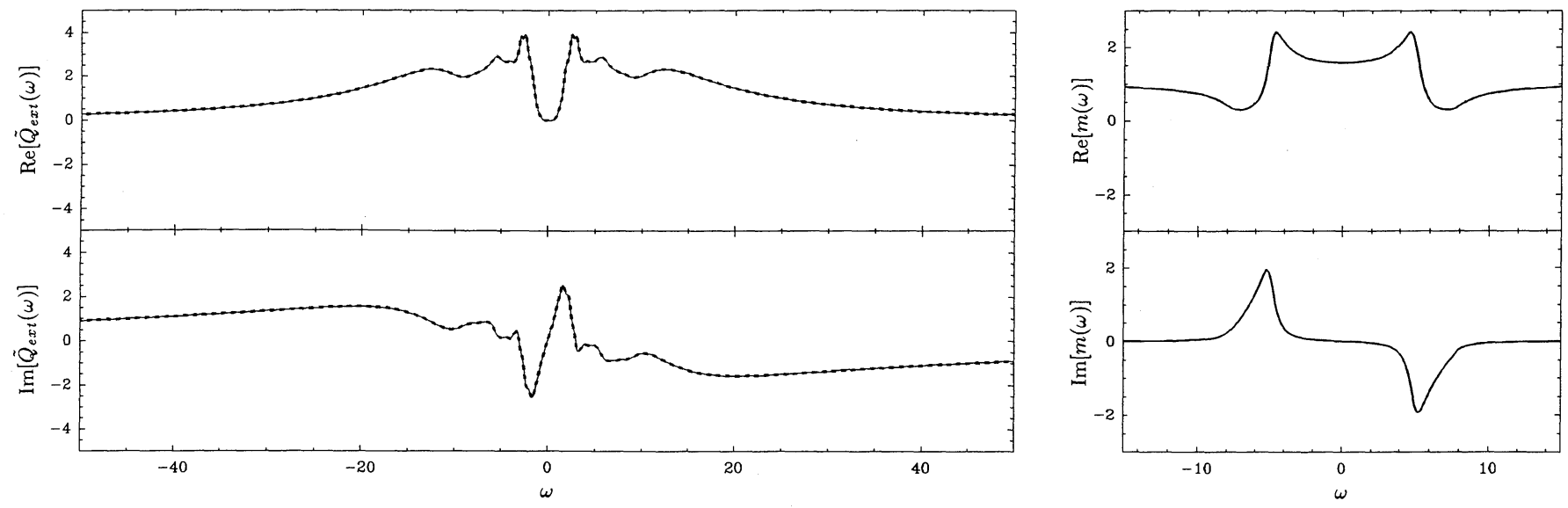

FIG. $1 a$

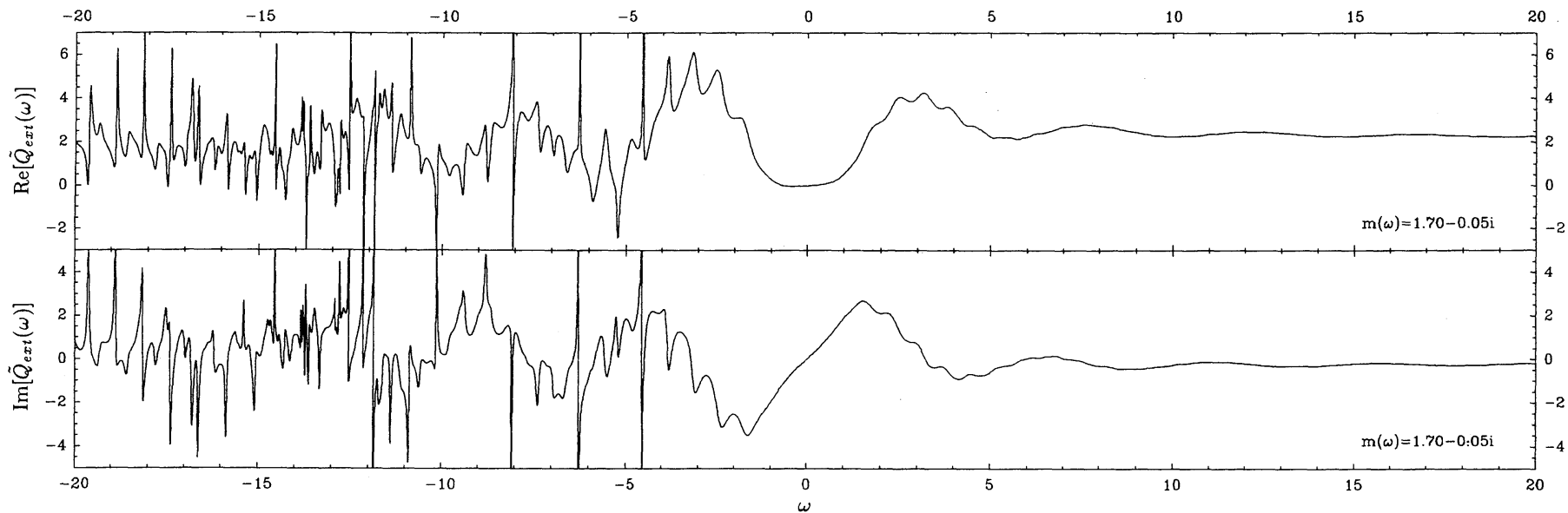

Fig. $1 b$

Fig. 1. $-(a) \tilde{Q}_{\text {ext }}(\omega)$ for a physical $m(\omega)$ given by eq. (15). Solid curves: the original $\widetilde{Q}_{\text {exx }}(\omega)$; dashed curves: respective numerical Hilbert transforms of $\widetilde{Q}_{\text {ext }}(\omega)$. The real and imaginary parts are presented separately. $m(\omega)$ is illustrated in the right box. $(b) \mathscr{Q}_{\text {ext }}(\omega)$ for a "nonphysical" $m(\omega)=1.7-0.05 i$.

fraction, in which $m(\omega)-1$ satisfies the Kramers-Kronig relations (7),

$$
m(\omega)=\sqrt{\epsilon(\omega)}=\left(1+\frac{\omega_{p}^{2}}{\omega_{0}^{2}-\omega^{2}+i \gamma \omega}\right)^{1 / 2}
$$

with $\omega_{p}=6.0, \omega_{0}=5.0$, and $\gamma=1.0$. Figure $1 b$ is a similar plot for a refractive index

$$
m(\omega)=1.7-0.05 i,
$$

which is a complex constant over the entire frequency range, so that $m(\omega)-1$ does not satisfy the Kramers-Kronig relations, and thus $m(\omega)$ is not physically correct. For the former case, we see that $\widetilde{Q}_{\text {ext }}(\omega)$ satisfies the crossing condition (6). Numerical computations clearly show in Figure $1 a$ that it fulfills the Hilbert transforms (4) as well. Within numerical accuracy there is an exact coincidence between the original and the transformed curves. (For a detailed description of the numerical Hilbert transforms technique, see the next section.) On the contrary, when $m(\omega)$ is "nonphysical" as assumed in equation (16), it is obvious that the crossing condition (6) is totally disobeyed and the asymptotic limit becomes 2 instead of 0 . Consequently the dispersion relations (14) also could not hold.

An obvious indication of these two numerical examples is that, in the angular frequency domain, a physically correct $\widetilde{Q}_{\text {ext }}(\omega)$ requires a physically correct $m(\omega)$. This is understandable if we notice that both $\widetilde{Q}_{\text {ext }}(\omega)$ and $m(\omega)$ [or $\left.\epsilon(\omega)\right]$ are employed to describe the electromagnetic response of a continuous medium, in the sense that the Kramers-Kronig expression for the complex $m(\omega)$ of the material of the particles in a medium is equivalent to a Kramers-Kronig representation of the effective refractive index of the medium consisting of independent particles. Hence, one may argue that $\widetilde{Q}_{\text {ext }}(\omega)$ is as basic as $m(\omega)$ [or $\epsilon(\omega)]$.

4. ANALYTICITY OF THE COMPLEX EXTINCTION EFFICIENCY IN THE SIZE PARAMETER DOMAIN: HOMOGENEOUS SPHERES

In this section we will explore the analyticity properties of $\tilde{Q}_{\text {ext }}$ for homogeneous spheres in the size parameter domain. Since now $G=\pi a^{2}$ ( $a$ is sphere radius), the complex extinction efficiency introduced in equation (11) becomes

$$
\tilde{Q}_{\mathrm{ext}}(x)=\frac{4}{x^{2}} S\left(0^{\circ}\right) \text {. }
$$

Here we have considered $\widetilde{Q}_{\text {ext }}$ as a function of the size parameter $x=k a=\omega a / c$ instead of the angular frequency $\omega$. This means we fix on a constant frequency and inquire how $\widetilde{Q}_{\text {ext }}$ 
varies as $x$ changes. Since the refractive index $m$ depends only on the light frequency, a fixed $\omega$ implies a fixed $m$.

From the discussions carried out at the end of the last section, one can expect that $\widetilde{Q}_{\text {ext }}(\tilde{x})$ in the $\tilde{x}$ domain will not have the same properties as $\tilde{Q}_{\text {ext }}(\tilde{\omega})$ in the $\tilde{\omega}$ domain. The reason for this is that, although the proportionality of $x$ to $\omega$ does not affect the analyticity properties of $\widetilde{Q}_{\text {ext }}$ when it goes from the $\tilde{\omega}$ to $\tilde{x}$ domain, the physical incorrectness of $m(\omega)=$ constant will destroy the causality of $\tilde{Q}_{\text {ext }}(x)$ completely. This was seen in Figure $1 b$. Thus the causality approach used for $\widetilde{Q}_{\text {ext }}(\omega)$ is no longer applicable to $\widetilde{Q}_{\text {ext }}(x)$.

Fortunately the Titchmarsh theorem offers us another option for studying the analyticity, that is, verifying if $\widetilde{Q}_{\text {ext }}(x)$ fulfills the Hilbert transforms. We have not been able to do this purely analytically because $\widetilde{\widetilde{Q}}_{\text {ext }}$ has no simple explicit expression. But we have advanced computer codes which can be used to calculate $\widetilde{Q}_{\text {ext }}$ at specified points, so as to verify numerically the satisfying of the Hilbert transforms.

Before implementing the numerical approach, we must modify $\widetilde{Q}_{\text {ext }}(x)$ somewhat in order to ensure that it has the proper asymptotic behavior (5) at large $x$ as required by the Titchmarsh theorem. Recalling the kinds of arguments we made in previous sections, it is clear that the quantity $\widetilde{Q}_{\text {ext }}(x)$ -2 will fulfill our purpose. Since 2 is the limit value of the diffraction theory approximation, $\widetilde{Q}_{\text {ext }}(x)-2$ approaches 0 as $|x| \rightarrow \infty$, so that we must apply numerical Hilbert transforms to $\widetilde{Q}_{\text {ext }}(x)-2$ rather than to $\widetilde{Q}_{\text {ext }}(x)$ itself.

In practice, for a specified refractive index $m$, we apply numerical Hilbert transforms to the real and imaginary parts of $\widetilde{Q}_{\text {ext }}(x)-2$ separately, namely,

$$
\begin{aligned}
& H_{i}\left(x_{0}\right)=-\frac{1}{\pi} P \int_{-\infty}^{+\infty} \frac{\operatorname{Im}\left[\tilde{Q}_{\mathrm{ext}}(x)-2\right]}{x-x_{0}} d x, \\
& H_{r}\left(x_{0}\right)=+\frac{1}{\pi} P \int_{-\infty}^{+\infty} \frac{\operatorname{Re}\left[\tilde{Q}_{\mathrm{ext}}(x)-2\right]}{x-x_{0}} d x .
\end{aligned}
$$

Then $H_{i}(x)$ and $H_{r}(x)$ are to be compared with $\operatorname{Re}\left[\widetilde{Q}_{\text {ext }}(x)-2\right]$ and $\operatorname{Im}\left[\widetilde{Q}_{\text {ext }}(x)-2\right]$, respectively, so that the analyticity of $\widetilde{Q}_{\text {ext }}(\tilde{x})$ in the lower $\tilde{x}$-plane is inferred from the possible differences.

From the computational point of view, the integrals in equations (17a) and (17b) are both of the type

$$
P \int_{-\infty}^{+\infty} \frac{g(x)}{x-x_{0}} d x .
$$

Hence they can be computed by the same computer algorithm. Note, however, that since equation (18) is an infinite Cauchy principal value integral, some special treatment is needed.

At first, we split equation (18) into three separate parts,

$$
\begin{aligned}
P \int_{-\infty}^{+\infty} \frac{g(x)}{x-x_{0}} d x & =\int_{-\infty}^{-a} \frac{g(x)}{x-x_{0}} d x \\
& +P \int_{-a}^{+b} \frac{g(x)}{x-x_{0}} d x+\int_{+b}^{+\infty} \frac{g(x)}{x-x_{0}} d x
\end{aligned}
$$

where $a$ and $b$ are positive numbers. If we choose quite large values for $a$ and $b$, the integrations over both positive and negative tails can be readily estimated by using asymptotic expansions for $\widetilde{Q}_{\text {ext }}(x)-2$. In the literature accessible to us, we found approximate expressions only for pure dielectric (Nussenzveig \& Wiscombe 1980; Attard et al. 1986) ( $m=$ real) and metal (Senior 1983) $(m=\infty)$ spheres. For all intermediate cases, with $m$ neither real nor $\infty$, the asymptotic behavior at large $x$ has been assumed to have a negative power form: $A x^{-\alpha}$ for both real and imaginary parts of $\widetilde{Q}_{\text {ext }}(x)-2$ with different exponents $\alpha$. Experience tells us that if $a$ and $b$ are large enough, the tail integrals are mainly decided by the values of $\widetilde{Q}_{\text {ext }}(x)-2$ at the cutting points $x=-a$, $+\mathrm{b}$. Thus one must set $a$ and $b$ as large as possible in order to ensure the accuracy of the numerical infinite integration.

The middle term in equation (19) is expected to contribute the major part to the final transforms. Obviously the integrand diverges at $x_{0}$. Sophisticated ways have been devised to treat this singularity, and well-designed algorithms are now generally available in several scientific software packages. In our computation, a subroutine implemented for a finite Hilbert transform in NAG (1983) has been adopted to fulfill our purpose.

As can be expected, the success of the numerical Hilbert transforms (17) will greatly depend on the efficiency and correctness of the Mie program used for the evaluation of $\widetilde{Q}_{\text {ext }}(x)$, especially at a large size parameter. The one we employed in this study is based on the algorithm suggested by Wang \& van de Hulst (1991), which can reliably compute $\widetilde{Q}_{\text {ext }}(x)$ for $x$ as large as thousands when $m$ is moderate.

We have done the numerical Hilbert transforms (17) for a number of specified refractive indices. The transformed curves are plotted in Figures 2 and 3 along with their respective original (untransformed) ones in the range $-20 \leq x \leq 20$. The real and imaginary parts of $\widetilde{Q}_{\text {ext }}(x)-2$ are presented separately. These curves have been arranged in a sequence of increasing values of $-\operatorname{Im}(m)$ from 0 to 400 while $\operatorname{Re}(m)$ is fixed as 1.33 in Figure 2 and 1.70 in Figure 3. With this organization, the evolution of $\widetilde{Q}_{\text {ext }}(x)-2$ versus $m$ may be clearly viewed. In our numerical integration, an adaptive resolution on the size parameter $x$ has been used in order that certain very sharp features could show up clearly. For all the calculations we have done, the values of both $a$ and $b$ in equation (19) are set to be 1500 .

By examining these figures closely, one notices that

1. The variations of the real and imaginary parts of $\tilde{Q}_{\text {ext }}(x)$ -2 are correlated with each other. Whenever a feature of $\operatorname{Re}\left[\tilde{Q}_{\text {ext }}(x)-2\right]$ appears, there is a corresponding feature of $\operatorname{Im}\left[Q_{\text {ext }}(x)-2\right]$ appearing in the same $x$-range.

2. When $-\operatorname{Im}(m)$ increases from 0 to the extremely large value of $400, \widetilde{Q}_{\text {ext }}(x)-2$ undergoes a change agreeing precisely with its Hilbert transforms at both extremes but exhibiting differences in the intermediate range. It is, in fact, reasonable to believe that Hilbert transforms are strictly fulfilled by $\widetilde{Q}_{\text {ext }}(x)$ -2 only when $m=$ real constant or $m=\infty$.

3. As for the crossing condition (6), $\widetilde{Q}_{\text {ext }}(x)-2$ shows a similar varying pattern to point 2 . That is, only when $m=$ real or $m=\infty$, does the symmetry relation hold for $\widetilde{Q}_{\text {ext }}(x)-2$.

4. When $m$ is a finite complex constant, there exist many discrepancies between the transformed and the corresponding original curves, which show the following patterns: (a) discrepancies appear as oppositely directed features with respect to a common background; (b) the central position of these features are located only on the negative part of the $x$-axis.

With the help of the Titchmarsh theorem, we can infer the analyticity properties of $\widetilde{Q}_{\text {ext }}(\tilde{x})-2$ in the lower $\tilde{x}$-plane from the characteristics listed above. Most obviously, based on point 2 , we can say that $\widetilde{Q}_{\text {ext }}(\tilde{x})-2$, thus $\widetilde{Q}_{\text {ext }}(\tilde{x})$, has no poles in 


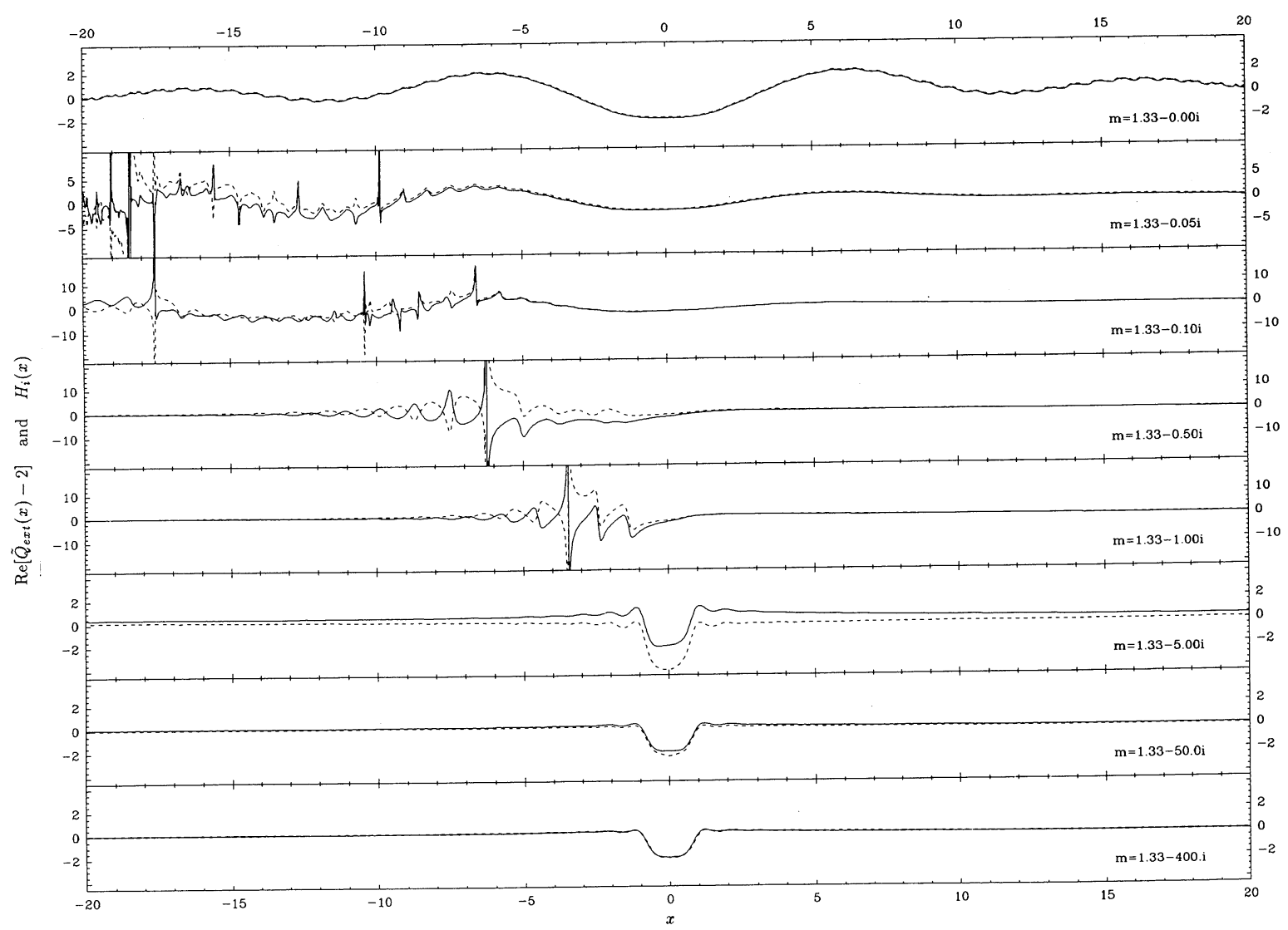

FIG. $2 a$

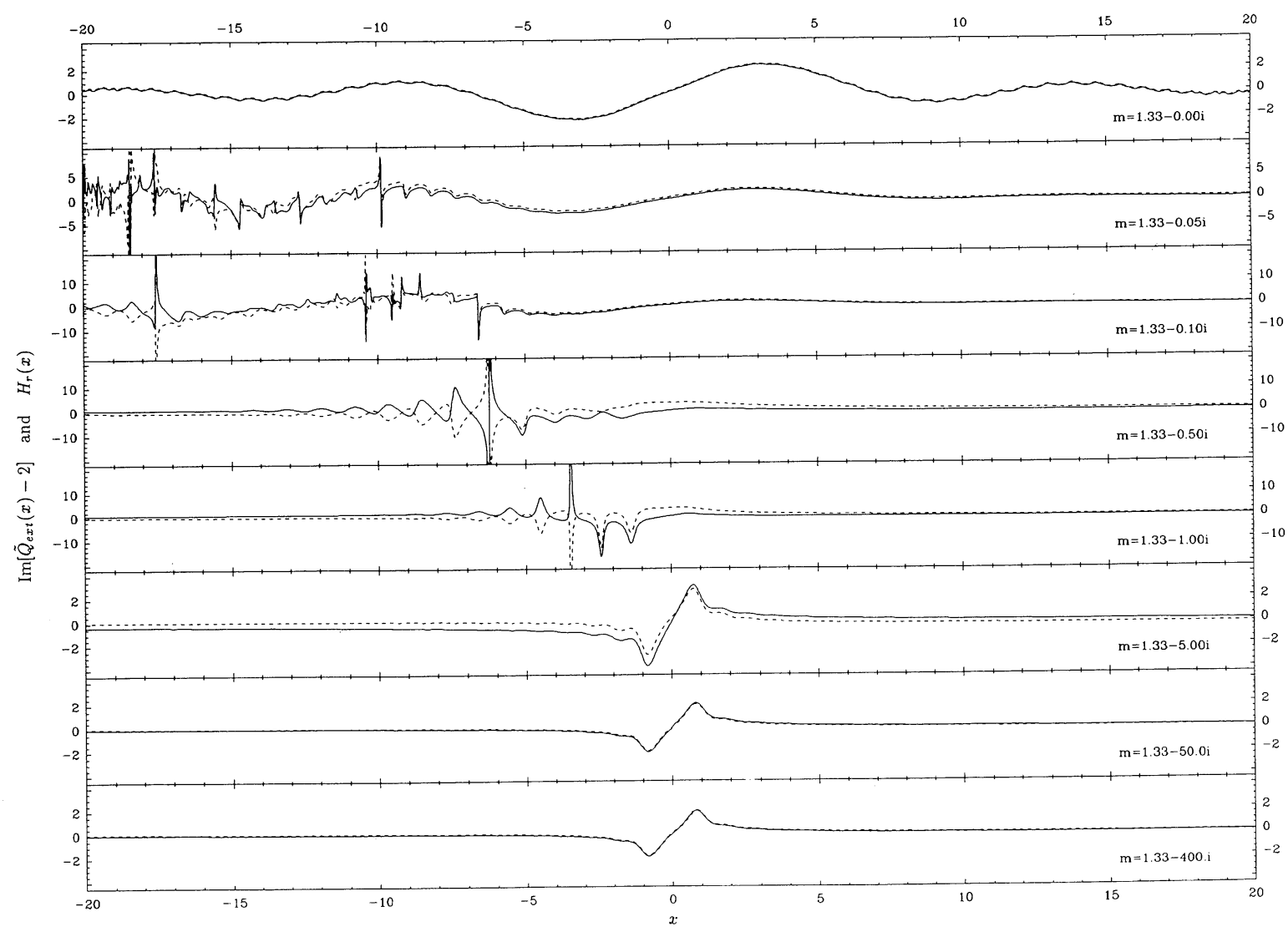

Fig. $2 b$

FIG. 2.-The original and respective numerical Hilbert transforms of $\widetilde{Q}_{\text {exil }}(x)-2$ for a sequence of $m$-values with $\operatorname{Re}(m)=1.33$. $(a)$ Solid lines: $\operatorname{Re}\left[\widetilde{Q}_{\text {exi }}(x)-2\right]$; dashed lines: $H_{i}(x)$ defined in eq. (17a). (b) Solid lines: $\operatorname{Im}\left[\widetilde{Q}_{\text {ext }}(x)-2\right]$; dashed lines: $H_{r}(x)$ defined in eq. $(17 \mathrm{~b})$. When $m$ takes intermediate values, $\widetilde{Q}_{\text {ext }}(x)$ has many very sharp features on the negative $x$-axis. Note that, for $x \geq 0, \operatorname{Re}\left[\tilde{Q}_{\text {ext }}(x)\right]$ is just the normal extinction curve. In order to let the main features show up fully different scales have had to be used. 

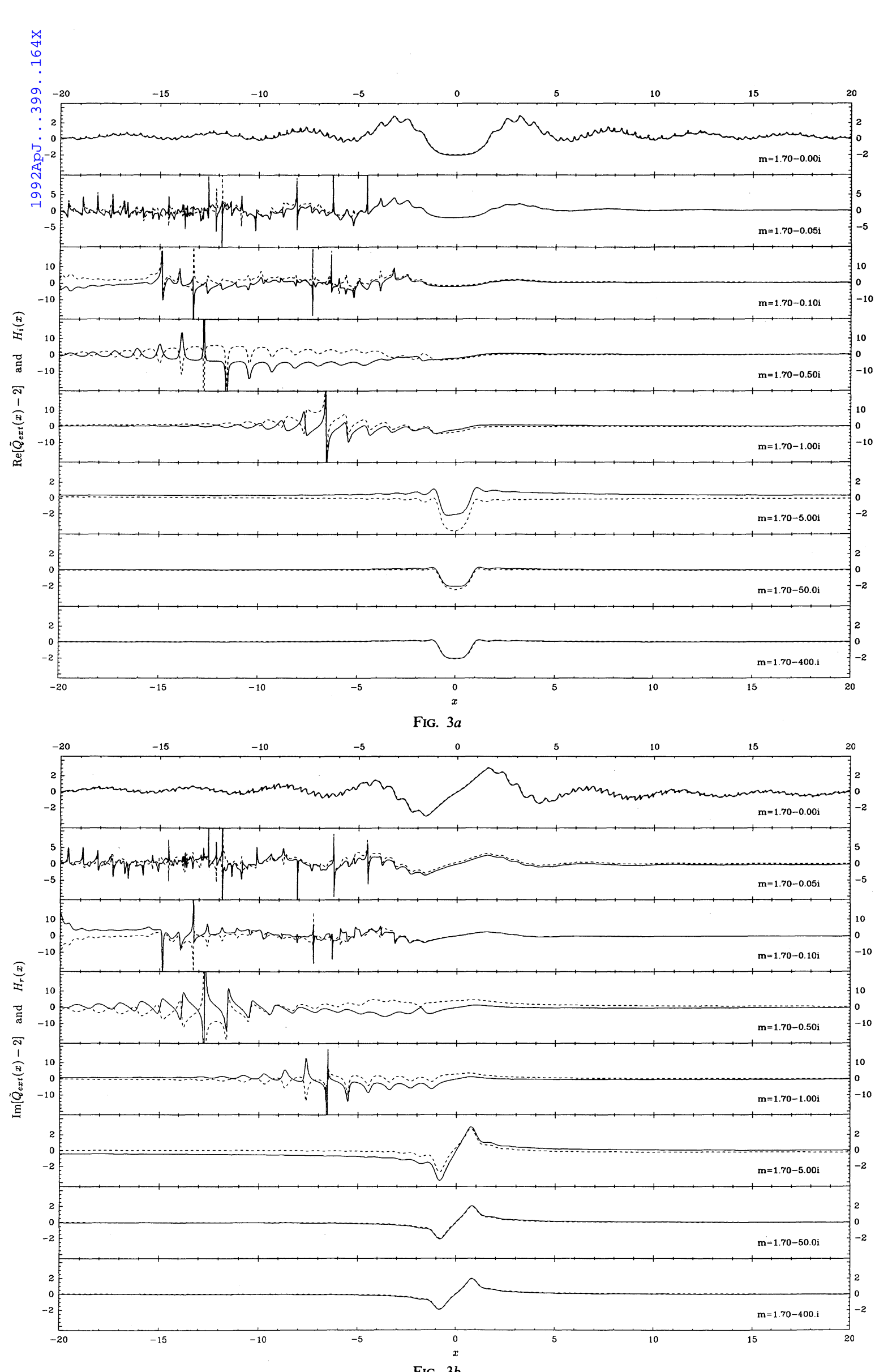

FIG. 3.-Similar to Fig. 2, but with $\operatorname{Re}(m)=1.70$ 

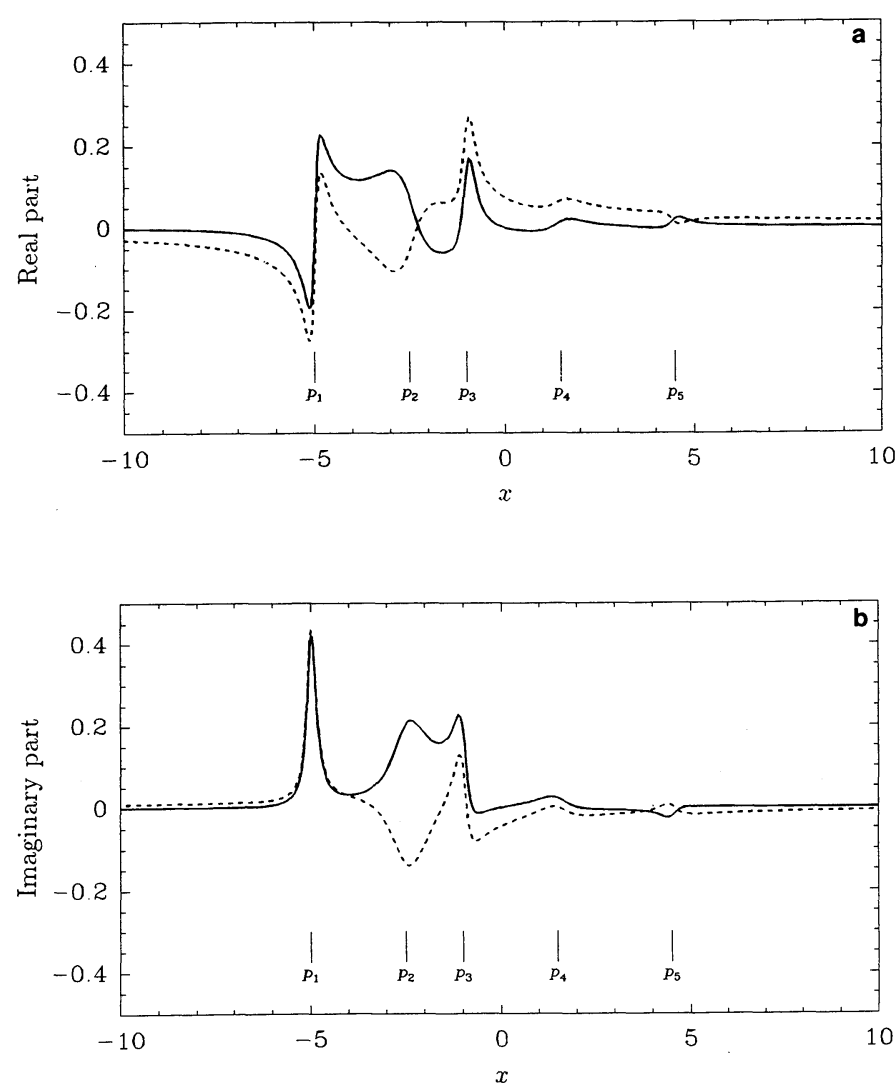

FIG. 4.-Inferring the poles of the rational function $F(\tilde{x})$ defined in eq. (20) by numerical Hilbert transforms. Solid lines: the original; dashed lines: respective numerical Hilbert transforms. (a) Real part of $F(\tilde{x}) ;(b)$ Imaginary part of $F(\tilde{x})$. The central positions of the poles are also indicated.

the lower $\tilde{x}$-plane if $m=$ real constant or $m=\infty$. Undoubtedly if $m$ is chosen as a finite complex constant, $\tilde{Q}_{\text {ext }}(\tilde{x})-2$ introduces poles in the lower $\tilde{x}$-plane because now the Hilbert transforms (4) are violated. This becomes obvious if we carry out the numerical integral transforms (17) on a much simpler rational function. As an example, consider

$$
F(\tilde{x})=\frac{\left(\tilde{x}-z_{1}\right)\left(\tilde{x}-z_{2}\right)}{\left(\tilde{x}-p_{1}\right)\left(\tilde{x}-p_{2}\right)\left(\tilde{x}-p_{3}\right)\left(\tilde{x}-p_{4}\right)\left(\tilde{x}-p_{5}\right)},
$$

with $z_{1}=0, z_{2}=3.0-1.0 i, p_{1}=-5.0+0.15 i, p_{2}=-2.5$ $-0.6 i, \quad p_{3}=-1.0+0.2 i, \quad p_{4}=1.5+0.5 i, \quad p_{5}=4.5-0.25 i$. The numerical results are shown in Figure 4. Clearly function (20) has three poles, $p_{1}, p_{3}$, and $p_{4}$, in the upper $\tilde{x}$-plane and two poles, $p_{2}$ and $p_{5}$, in the lower plane. We see that only $p_{2}$ and $p_{5}$ show up as locally reversed features in the transformed and the original curves. Furthermore the central positions of these discrepancies are decided by the real part of the poles, and the range over which a discrepancy appears is approximately proportional to the imaginary part of the corresponding pole. Based on this example and our point 4 , we may suggest that the possible poles of $\tilde{Q}_{\text {ext }}(\tilde{x})-2$, and, of course, $\tilde{Q}_{\text {ext }}(\tilde{x})$, which are in the lower $\tilde{x}$-plane, are only limited to the left side. In the next section, we will see that it is this interesting mathematical phenomenon that validates the approximation method proposed there.

It would be very gratifying if our assertions above could be proved strictly rather than numerically. We even suspect that such a proof may have been given by somebody some years ago, since Mie's result has been known for such a long time, but we have not been able to find it. However, our numerical approach is always helpful and actually offers a pragmatic method for studying analyticity, especially when we have no simple explicit expressions for the functions under investigation.

\section{APPROXIMATING THE CALCULATION OF MEAN EXTINCTION}

From now on, we shift our discussion from the complex size parameter $\tilde{x}$ domain to the complex particle size $\tilde{a}$ domain. Because $x=k a$, it is obvious that the conclusions we have drawn about $\widetilde{Q}_{\text {ext }}(\tilde{x})$ in the $\tilde{x}$-plane carry over for $\widetilde{Q}_{\text {ext }}(k \tilde{a})$ in the complex $\tilde{a}$-plane. Here a constant angular frequency $\omega$ is assumed as before, which means the wavenumber $k=2 \pi /$ $\lambda=\omega / c$ is a real number. We shall restate these conclusions for $\tilde{Q}_{\text {ext }}(k \tilde{a})$ in the complex $\tilde{a}$ domain for future reference. When $m$ is real or $\infty, \widetilde{Q}_{\text {ext }}(k \tilde{a})$ is analytic in the entire lower $\tilde{a}$-plane. But as $m$ becomes complex, poles are introduced in the lower $\tilde{a}$-plane, which are, however, limited to the left side. Furthermore $\widetilde{Q}_{\text {ext }}(k a)$ approaches the limit value 2 as $|a|$ becomes very large,

$$
\lim _{|a| \rightarrow \infty} \tilde{Q}_{\mathrm{ext}}(k a)=2 .
$$

We shall now reconsider the problem of evaluating the mean extinction introduced in $\S 1$.

First, consider the complex integral

$$
\oint \pi \tilde{a}^{2} \tilde{Q}_{\mathrm{ext}}(k \tilde{a}) n(\tilde{a}) d \tilde{a}
$$

over the contour $C$ shown in Figure 5. Here $n(\tilde{a})$ denotes an appropriate complex function which approximates our actual size distribution when $\tilde{a}$ is real ${ }^{2}$ and it has a "limited" number of discrete simple poles in the lower $\tilde{a}$-plane. We further require that $n(\tilde{a})$ approach zero sufficiently fast when $|\tilde{a}| \rightarrow \infty$, namely,

$$
\lim _{|\tilde{a}| \rightarrow \infty} \tilde{a}^{3} n(\tilde{a})=0,
$$

so that the application of the residue theorem (Arfken 1985) to the contour integral equation (22) will result in

${ }^{2}$ This means that $n(\tilde{a})$ becomes a real function when $\tilde{a}$ is real and it approximates the actual size distribution in the entire real $a$-axis. Although an actual size distribution must be 0 for all $a<0$, the approximate $n(a)$ may not be zero when $a<0$; however, it should be negligibly small.

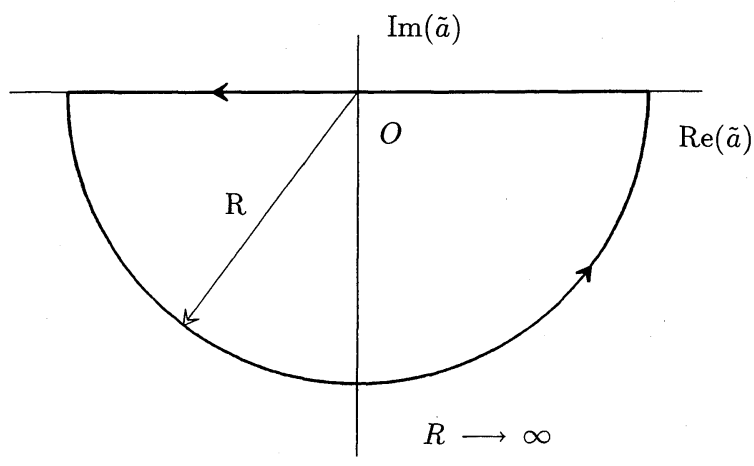

FIG. 5.-Contour $C$ used in complex integral (22) 


$$
\begin{aligned}
& \int_{-\infty}^{\infty} \pi a^{2} \widetilde{Q}_{\text {ext }}(k a) n(a) d a \\
& \quad=-2 \pi i\left\{\begin{array}{l}
\text { residues at the poles of both } \widetilde{Q}_{\text {ext }}(k \tilde{a}) \\
\text { and } n(\tilde{a}) \text { in the lower } \tilde{a} \text { plane }
\end{array}\right\} .
\end{aligned}
$$

We shall employ the real part of the left-hand side of equation (24) as an estimation of the mean extinction cross section defined in equation (1). This is reasonable because $n(a)$ is expected to be negligibly small when $a$ is negative. Therefore the integration over size distribution is reduced to an evaluation of the residues at discrete poles.

One may question the feasibility of conveniently finding such a complex function $n(\tilde{a})$. This suspicion can be dispelled if one notices that nonlinear rational approximation theory has become one of the well-established branches of numerical analysis during the past decades (Braess 1980). In fact one can rather accurately approximate most of the frequently used practical size distributions by rational functions (ratios of two polynomials) with only a few simple poles. Details on this matter will be presented in our subsequent papers.

Careful readers may also notice that on the right-hand side of equation (24), the residue contributions are not only from the poles of $n(\tilde{a})$, but also from these of $\widetilde{Q}_{\text {ext }}(k \tilde{a})$. From the discussions of $\S 4$, we know that the possible poles of $\widetilde{Q}_{\text {ext }}(k \tilde{a})$ in the lower left plane are enormous and their locations are complicated as well. These may at first seem to prevent a practical implementation of our approximation scheme as proposed, especially when $m$ is complex. However, the versatility of rational approximation theory allows us to construct $n(\tilde{a})$ in such a way that $n(a)$ approaches zero very quickly on the negative $a$-axis. Consequently $n(\tilde{a})$ also will vanish quickly in the entire left-hand $\tilde{a}$-plane. As a result, the residue contributions from all the possible poles of $\widetilde{Q}_{\text {ext }}(k \tilde{a})$ can be generally neglected, since these poles are only located in the left side and their effects are highly suppressed by the smallness of $n(\tilde{a})$ in the entire left $\tilde{a}$-plane. Hence, as will be numerically demonstrated, in the final evaluation, only the poles of $n(\tilde{a})$ will be involved.

Summarizing all the above arguments, we are left with the following approximation:

$$
\begin{aligned}
\int_{0}^{\infty} \pi a^{2} \tilde{Q}_{\mathrm{ext}}(a) & n(a) d a \\
& \approx-2 \pi i \sum_{p}\left\{\pi \tilde{a}_{p}^{2} \tilde{Q}_{\mathrm{ext}}\left(k \tilde{a}_{p}\right)\left[\left(\tilde{a}-\tilde{a}_{p}\right) n(\tilde{a})\right]_{\tilde{a}=\tilde{a}_{p}}\right\},
\end{aligned}
$$

where $\tilde{a}_{p}$ denotes the possible pole of $n(\tilde{a})$ in the lower $\tilde{a}$-plane, which should be of order of one. Note that the mean extinction defined in equation (1) is just the real part of equation (25).

The approximation given in equation (25) requires the computation of $\widetilde{Q}_{\text {ext }}(\tilde{x})$ for complex size parameter $\tilde{x}=k \tilde{a}$. This further requires modifying the usual Mie program so that a complex size parameter $\tilde{x}$ may be used as input. For most cases, this revision is not too difficult.

As already mentioned, the approximation neglects any possible residue contribution from the poles of $\tilde{Q}_{\text {ext }}(k \tilde{a})$. Seeing how these poles are distributed $(\S 4)$, it is rather difficult by strict analysis to estimate, a priori, the accuracy of our method. Direct numerical integration is the only way to check how good (or bad) our method is.

We have derived an explicit form of equation (25) using the size distribution,

$$
n(a)=\left(\frac{3}{2 \pi \beta}\right) /\left[\left(\frac{a-\alpha}{\beta}\right)^{6}+1\right],
$$

which is illustrated in Figure 6. Now equation (25) becomes

$$
\begin{aligned}
\int_{0}^{\infty} \pi a^{2} \tilde{Q}_{\mathrm{ext}}(k a) n(a) d a & \approx \frac{1-e^{-i(\pi / 3)}}{2} \pi\left[\tilde{a}_{0}^{2} \tilde{Q}_{\mathrm{ext}}\left(k \tilde{a}_{0}\right)\right. \\
& \left.+e^{-i(\pi / 3)} \tilde{a}_{1}^{2} \tilde{Q}_{\mathrm{ext}}\left(k \tilde{a}_{1}\right)+e^{-i(2 \pi / 3)} \tilde{a}_{2}^{2} \tilde{Q}_{\mathrm{ext}}\left(k \tilde{a}_{2}\right)\right]
\end{aligned}
$$

with three poles located in the lower plane at

$$
\tilde{a}_{0}=\alpha+\beta e^{-i(\pi / 6)}, \quad \tilde{a}_{1}=\alpha+\beta e^{-i(\pi / 2)}, \quad \tilde{a}_{2}=\alpha+\beta e^{-i(5 \pi / 6)} \text {. }
$$

Numerical examples are calculated for $\alpha=1.0$ and $\beta=0.5$. Two sets of curves have been plotted (Figs. 7 and 8). Each one corresponds to a fixed $\operatorname{Re}(m)$. Results obtained by direct numerical integration are represented by thin solid lines, and the residue evaluations by thick dashed lines. The differences are shown as thin dashed lines. For clarity, the real and imaginary parts are illustrated separately.

These examples show that our approximation is really quite good. For all the values of $m$ we have tried, the absolute differences between direct integration and the residue approximation are less than $1 \%$. The mean relative error is only about $0.5 \%$ for a large range of $k$ (from 0 to 10 ). Such a small error exceeds one's expectation when $m$ is complex. Looking back at Figures 2 and 3, we can see that quite large differences exist between the numerically Hilbert-transformed and the original $\widetilde{Q}_{\text {ext }}(x)-2$, especially for $0.1<-\operatorname{Im}(m)<50$. But if we recall that the analyticity is a localized property and the integrand of equations (25) or (27) approaches 0 with a speed faster than $O\left(a^{2}\right)$ when $a \rightarrow 0$, this high accuracy becomes reasonable. We have performed many other calculations for different size distributions and refractive indices. All show a similar high accuracy.

\section{SUMMARY AND DISCUSSION}

In previous sections, we have explored the analyticity of the complex extinction efficiency $\widetilde{Q}_{\text {ext }}$ in different parameter domains. By employing the physical concept of causality, we found that $\widetilde{Q}_{\text {ext }}(\tilde{\omega})$ is analytic in the entire lower $\tilde{\omega}$-plane. And a

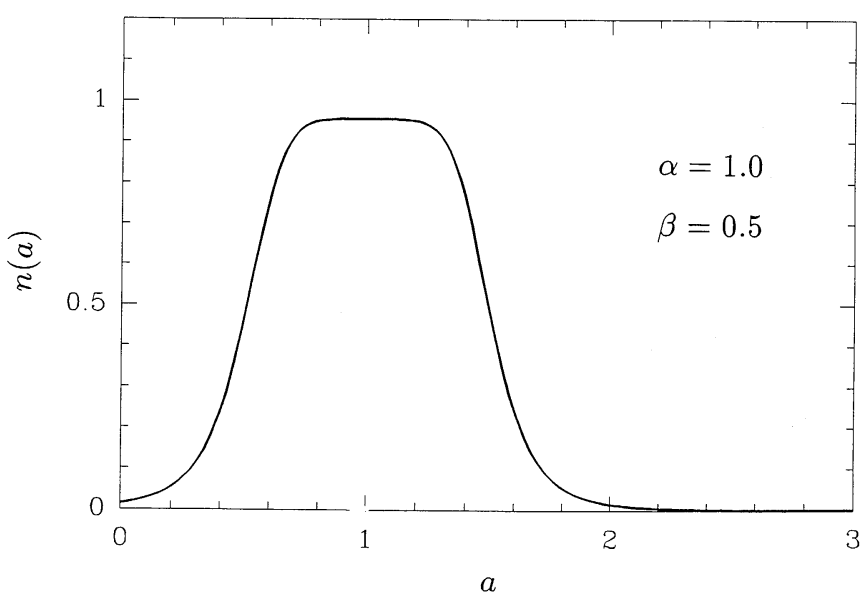

FIG. 6.-Size distribution $n(a)$ defined in eq. (26). It is normalized as $\int_{-\infty}^{+\infty} n(a) d a=1$. Note that, with the given parameters, it has a very small tail on the negative $a$-axis. 

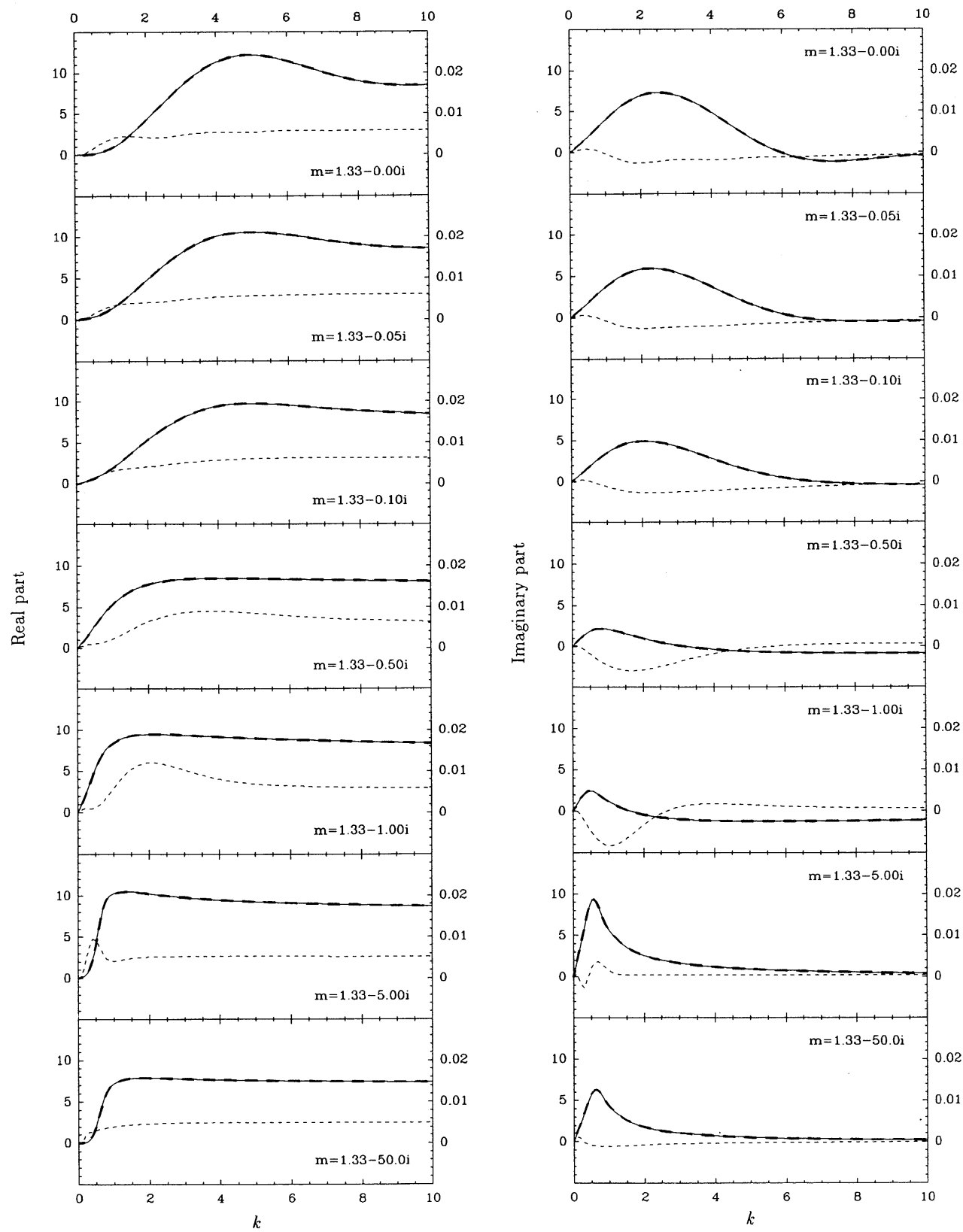

FIG. 7.-Comparison between direct integration and the residue approximation for a sequence of values of $m$ with $\operatorname{Re}(m)=1.33$. Thin solid lines: direct numerical integration of the left-hand side of eq. (27); thick long dashed lines: residue calculation by the right-hand side of eq. (27); thin short dashed lines: difference = residue approximation - direct integration. (a) Real parts; (b) Imaginary parts. Notice that the scale for the differences as labeled on the right side is 500 times the scale of the mean cross section. For each curve, 100 points have been plotted.

few simple physical arguments show that $\widetilde{Q}_{\text {ext }}(\omega)$ must vanish when $|\omega|$ goes to infinity. All of these strongly indicate that the causality of $\widetilde{Q}_{\text {ext }}(\omega)$ implies the causality of $m(\omega)-1$, or vice versa. We think this is not just a mathematical coincidence. Instead it comes from the self-consistency of Maxwell theory.

The major part of this Paper has been devoted to the discussion of $\widetilde{Q}_{\text {ext }}(\tilde{x})$ in the complex size parameter $\tilde{x}$ domain. In the $\tilde{x}$-plane, $\tilde{Q}_{\text {ext }}$ behaves very differently than in the complex $\tilde{\omega}$-plane. The most prominent difference is that $\tilde{Q}_{\text {ext }}(x)$ approaches the limit 2 when $x$ goes to infinity. Even though poles appear in the lower $\tilde{x}$-plane when the refractive index $m$ is complex, all of our carefully calculated examples suggest that these poles are located only on the left side. Although we are still unable to strictly (analytically) prove these results, we believe they are generally mathematical truths. In this Paper we studied only the case of homogeneous spheres. At the next stage we will extend our investigations to other particle shapes and configurations.

Our detailed investigations of the analyticity of $\tilde{Q}_{\text {ext }}(\tilde{x})$ have resulted in a powerful approximation, which promises to reduce greatly the evaluation of mean extinctions. Besides its practical usefulness, this approximation method releases us from the narrower positive $x$-axis to a much wider complex $\tilde{x}$-plane. We hope that such an extension will be helpful for the 

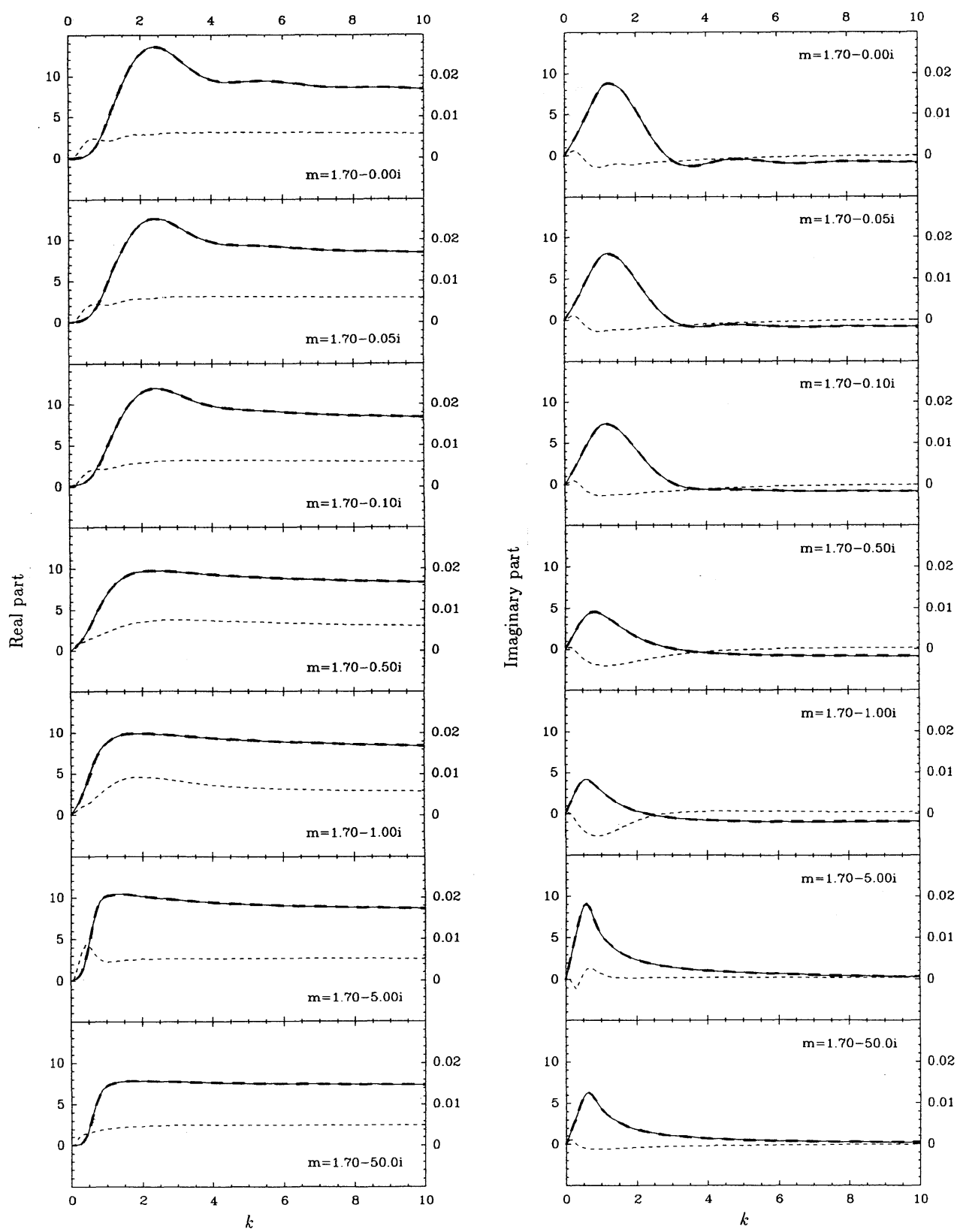

FIG. 8.-Similar to Fig. 7, but with $\operatorname{Re}(m)=1.70$

solving of other related problems, such as particle sizing. As we have already seen, a complex extinction efficiency with a complex $\tilde{x}$ reveals hitherto unexpected properties. We suspect that much more information remains to be discovered in the complex $\tilde{x}$-plane. The advantage of the approximation method derived here will become even more obvious when the particles have nontrivial shapes (say spheroids). And future development of useful expression of a given $n(a)$ in terms of rational function or other functions having simple poles is also needed. We will address these problems in our forthcoming papers.

Finally we have learned from our investigation that numerical Hilbert transforms are practical and efficient tools for the studying of the analytic properties of complex functions. In fact, by using these and other similar transforms one can infer the analiticity of a complex function in any area of the complex plane. Analogously, through the differences shown by the transformed and the corresponding original functions, we can gain a general view about the distribution of the poles (or zeroes) in the entire complex plane.

Z. F. X. would like to acknowledge an assistantship offered by Huygens Laboratory of Leiden University and a scholarship awarded by the World Laboratory. We thank J. I. Hage for his kindness in allowing us to use his excellent Mie program. We are grateful to $\mathbf{H}$. C. van de Hulst and C. V. M. van der Mee for carefully reading the manuscript and making useful suggestions. We are indebted to W. Chotkowski for helping with the computer applications. We also acknowledge the interesting comments made by C. F. Bohren and P. G. Martin. 


\section{REFERENCES}

Arfken, G. 1985, Mathematical Methods for Physicists (3d ed.; New York: Academic)

IA Asano, S., \& Yamamoto, G. 1975, Appl. Opt., 14, 29

IO' Attard, P., Box, M. A., Bryant, G., \& McKellar, B. H. J. 1986, J. Opt. Soc. Am. $\lim _{i \rightarrow 1} \mathrm{~A}, 3,256$

Bode, H. W. 1945, Network Analysis and Feedback Amplifier Design (New York: Van Nostrand)

Bohren, C. F., \& Huffman, D. R. 1983, Absorption and Scattering of Light by Small Particles (New York: John Wiley)

Braess, D. 1980, Nonlinear Approximation Theory (Berlin: Springer)

Greenberg, J. M. 1960, J. Appl. Phys., 31, 82

Hage, J. I., Greenberg, J. M., \& Wang, R. T. 1991, Appl. Opt., 30, 1141

Kerker, M. 1969, The Scattering of Light and Other Electromagnetic Radiation (New York: Academic)

Ku, J. C., \& Felske, J. D. 1986, J. Opt. Soc. Am. A, 3, 617

NAG FORTRAN Library Manual, Mark 10, 1983, Numerical Algorithms Group, Oxford
Newton, R. G. 1966, Scattering Theory of Waves and Particles (New York: McGraw-Hill)

Nussenzveig, H. M. 1972, Causality and Dispersion Relations (New York: Academic)

Nussenzveig, H. M., \& Wiscombe, W. J. 1980, Phys. Rev. Lett., 45, 1490

Purcell, E. M. 1969, ApJ, 158, 433

Purcell, E. M., \& Pennypacker, C. R. 1973, ApJ, 186, 705

Senior, T. B. A. 1983, Appl. Opt., 22, 1796

Titchmarsh, E. C. 1937, Introduction to the Theory of Fourier Integrals (Oxford: Clarendon)

Toll, J. S. 1956, Phys. Rev., 104, 1760

van de Hulst, H. C. 1957, Light Scattering by Small Particles (New York: Dover)

van Kampen, N. G. 1953, Phys. Rev., 89, 1072

Wang, R. T., \& van de Hulst, H. C. 1991, Appl. Opt., 30, 106 